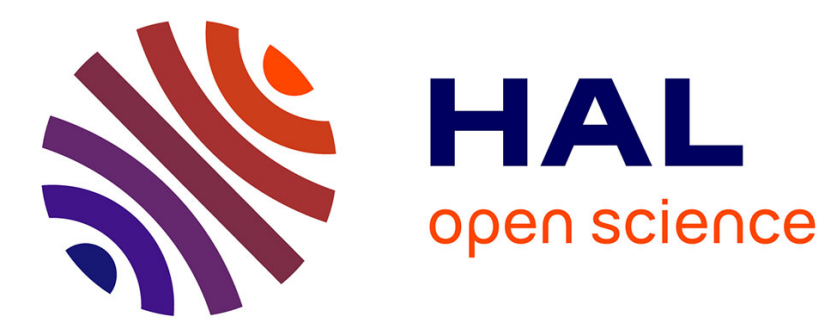

\title{
Consistent State Estimation on Manifolds for Autonomous Metal Structure Inspection
}

Bryan Starbuck, Alessandro Fornasier, Stephan Weiss, Cédric Pradalier

\section{To cite this version:}

Bryan Starbuck, Alessandro Fornasier, Stephan Weiss, Cédric Pradalier. Consistent State Estimation on Manifolds for Autonomous Metal Structure Inspection. ICRA 2021, Feb 2021, virtual event, China. IEEE Computational Science and Engineering, 2021, 10.1109/ICRA48506.2021.9561837. hal-03445976

\section{HAL Id: hal-03445976 \\ https://cnrs.hal.science/hal-03445976}

Submitted on 6 Dec 2021

HAL is a multi-disciplinary open access archive for the deposit and dissemination of scientific research documents, whether they are published or not. The documents may come from teaching and research institutions in France or abroad, or from public or private research centers.
L'archive ouverte pluridisciplinaire HAL, est destinée au dépôt et à la diffusion de documents scientifiques de niveau recherche, publiés ou non, émanant des établissements d'enseignement et de recherche français ou étrangers, des laboratoires publics ou privés. 


\title{
Consistent State Estimation on Manifolds for Autonomous Metal Structure Inspection
}

\author{
Bryan Starbuck ${ }^{1 \dagger}$, Alessandro Fornasier ${ }^{2 \dagger}$, Stephan Weiss ${ }^{2}$, and Cédric Pradalier ${ }^{1}$
}

\begin{abstract}
This work presents the Manifold Invariant Extended Kalman Filter, a novel approach for better consistency and accuracy in state estimation on manifolds. The robustness of this filter allows for techniques with high noise potential like ultra-wideband localization to be used for a wider variety of applications like autonomous metal structure inspection. The filter is derived and its performance is evaluated by testing it on two different manifolds: a cylindrical one and a bivariate b-spline representation of a real vessel surface, showing its flexibility to being used on different types of surfaces. Its comparison with a standard EKF that uses virtual, noise-free measurements as manifold constraints proves that it outperforms standard approaches in consistency and accuracy. Further, an experiment using a real magnetic crawler robot on a curved metal surface with ultra-wideband localization shows that the proposed approach is viable in the real world application of autonomous metal structure inspection.
\end{abstract}

\section{INTRODUCTION}

Routine inspection of large metal structures is of the utmost importance in avoiding environmental catastrophe and maintaining safety standards. Small differential-drive robots with magnetic wheels are being deployed on vessels and cargo ship hulls to ensure that these standards are met, but as of yet, the task is being completed via manual operation. Given the expansive dimensions of these structures, completing this task autonomously would be preferable, but with such high stakes, having the best localization accuracy and consistency is paramount. Even though classical methods for state estimation exist, they do not consider the fact that the robot is a planar robot moving on a curved surface. Thus, they tend to estimate the six-dimensional state, enforcing constraints on all known degrees of freedom, affecting the consistency of the approach. Therefore, motivated by the recent development of the consistent Invariant Extended Kalman Filter (IEKF) [1] [2] [3] [4], in this work, we propose a Manifold Invariant Extended Kalman Filter, a novel approach to consistent state estimation on manifolds with application to ship hull inspection.

A metal structure, e.g. a ship hull, can be thought of as a smooth surface embedded in three-dimensional Euclidean

This work was supported by the EU-H2020 project BUGWRIGHT2 (GA 871260)

${ }^{1}$ Bryan Starbuck and Cédric Pradalier are with Georgia Tech Lorraine - CNRS UMI 2958 bstarbuck3@gatech.edu, cedric.pradalier@georgiatech-metz.fr

${ }^{2}$ Alessandro Fornasier and Stephan Weiss are with the Control of Networked Systems Group, University of Klagenfurt, Austria \{alessandro.fornasier, stephan.weiss\}@ieee.org

${ }^{\dagger}$ These authors contributed equally

Accepted February/2021 for ICRA 2021, DOI follows ASAP OIEEE.

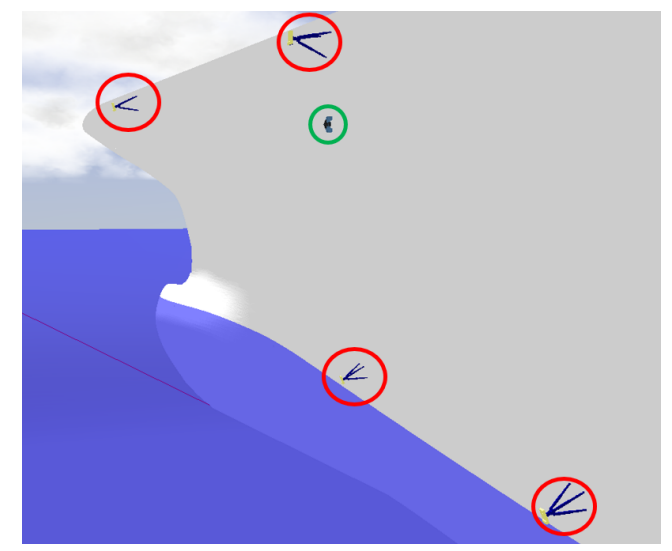

Fig. 1. Simulation of a Magnetic Crawler robot (green circle) on a Ship Hull with an Ultra-wideband Localization Grid (red circles).

space, which can be viewed as a two-dimensional, differentiable, Riemannian manifold. This allows us to select a chart from the maximum atlas and hence define a chart map, a continuous, invertible, bijective map, that maps each point of the considered manifold to a two-dimensional Euclidean space. The full state of the planar robot moving on the manifold is six-dimensional, including position and orientation within a three-dimensional euclidean space, however, its "planarity" gives only three degrees of freedom: a two-dimensional position and the heading angle. Therefore, by applying a consistent IEKF on the product space $\mathbb{R}^{2} \times S O(2)$, the localization problem can be solved entirely on the chart.

Being able to evaluate the surface and it's derivatives at any point is necessary to create a basis for the tangent space in order to recover the full orientation of the robot from the minimal state estimated on the chart. Given that there are no equations for generic metal structures like a ship hull, and that the equation of a surface must be known to apply the proposed methodology, a bivariate b-spline representation of the surface was recognized as a sufficient substitute. This can be obtained by extracting the vertices of the surface from its CAD model for interpolation, or by taking a laser scan of the structure and interpolating the resulting point cloud.

The propagation model of the magnetic crawler robot is based on its odometry measurements [5], but even with high precision wheel encoders, this is only reliable for predicting the robot's state within a plane that is tangent to the surface. The measurement model for state localization is given by modelling ultra-wideband (UWB) range measurements with a trilateration framework [6], which in ideal conditions can accurately update the robot's position within $\pm 5 \mathrm{~cm}$, but with high noise potential from wave deflection off the metal 
surface, this is not a safe assumption to make [7]. Therefore, to account for the inherent drifting from the surface that the robot's state will experience, classical approaches tend to solve the localization problem by forcing constraints within the Extended Kalman Filter (EKF) framework [8]. Imposing two virtual, zero noise measurements as constraints such that the first constraint maps the state of the robot to the surface, and the second one maintains collinearity between the vertical axis of the robot and the normal to the surface resulting in a full estimation of the robot's position and orientation, but sacrificing the filter's consistency. With the loss of consistency, the loss of accuracy and robustness follows. To validate the benefit and versatility of the proposed approach compared to classical approaches, simulations were carried out specifically on cylindrical and curved surfaces, simulating respectively a cylindrical vessel and a ship hull. Moreover, An experiment with a real magnetic crawler robot on a curved metal plate has been performed to show the feasibility of the methodology in real-world scenarios.

\section{RELATED WORK}

Strategies for metal structure inspection can take on many forms, but in every case, fundamental questions must be investigated, such as: Which sensors should be used for mapping and localization? and, Which filtering technique will produce the best results? For bridge inspection, unmanned aerial vehicles (UAVs) equipped with lidar for mapping and visual, inertial odometry systems for localization collect data from the bridge to be processed for structural analysis [9]. For ship hull inspection, autonomous underwater vehicles (AUVs) equipped with cameras for mapping and sonar systems for localization similarly complete the task [10]. However, it should be noted that the inspection of metal structures and vessels is not solely confined to airborn inspection or to below the waterline. In fact, large cargo ships can protrude up to and exceeding fifty meters above water level especially when unloaded. Therefore, to complete the inspection most efficiently and in it's entirety, utilizing a combination of UAVs, AUVs, and differential-drive, magnetic-wheeled crawler robots could be quite advantageous.

The crawlers hold primary responsibility for inspecting the portion of the ship hull that protrudes from the water, and high accuracy localization is fundamental to this being accomplished autonomously. There are various sensors that come to mind as candidates for correcting the position of the robot such as RTK-GPS, Wifi, and UWB. RTK-GPS is too unreliable given that clear line of sight to satellites is always required, and Wifi is also unreliable because it is too sensitive to interference. UWB which is based on the time of flight of wave transmission resulting in a range measurement is proven to be a reliable method of localizing multiple moving targets [11]. The major factor which highlights UWB as a more robust method for this application is that it has high bandwidth meaning that the waves experience less interference while reliably transmitting small packets of data at a distance generally up to 30 meters [12]. Although UWB is generally used for indoor object tracking, given that more specialized filters are being developed to enhance its robustness, it is becoming increasingly feasible to experiment

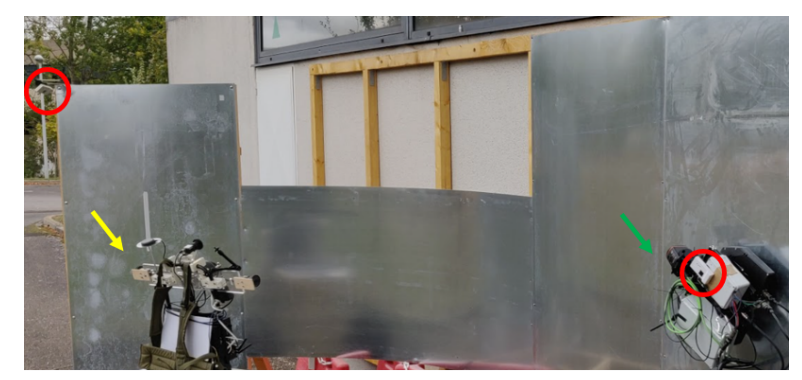

Fig. 2. Magnetic Crawler Robot (green arrow) on a Curved Metal Surface with Ultra-wideband Localization (red circles) and laser (yellow arrow) to track the robot for ground truth.

with outdoor applications like metal structure inspection. It therefore follows that a grid of UWB beacons for a robot to localize with respect to could be temporarily installed on the side of a ship hull. Fig. 1 shows a simulation of a ship hull with a magnetic crawler robot and four UWB beacons in place to form a localization grid.

Two main factors to consider when developing a filter for a problem like this are its accuracy and consistency. It can be difficult to maintain accuracy when using UWB for metal structure inspection due to high noise from wave deflection off the metal surface. This error causes a prolonged time of flight resulting in over-exaggerated range measurements. Some propose including methods of detecting these divergences by analysing the noise distribution to decide if a measurement is usable [13]. Others suggest loosely or tightly coupled filters to resolve the problem [14]. A loosely coupled, two step update of orientation correction followed by position correction can give good results, although it is said that a tightly coupled measurement model, where position and orientation are corrected at the same time can better overcome large positioning errors [15]. Even when using tightly coupled EKFs to achieve higher accuracy, there is still the likelihood of inconsistency in this case due to the aforementioned problem related to the robot's planarity being expressed with six degrees of freedom. This can cause the covariance of the robot's state to become disproportionately small resulting in an overconfidence in the propagation and eventually a divergence to an incorrect solution [16]. As Manifold filters solve this problem, they have proven themselves to be more consistent, and more accurate on average, than other filters [17]. The Invariant filter formulation [1] [2] [3] is proven to solve the aforementioned problems by ensuring the Log-Linear property of the error, that is, the independence of the error dynamics from the state estimate. We employed the Invariant filter formulation within a manifold-based space showing that our Manifold Invariant Extended Kalman Filter (M-IEKF) results in greater consistency and improved accuracy.

\section{THEORY}

In this section, a general understanding of differential geometry, manifolds, and bivariate b-spline surface representations is introduced.

\section{A. Manifolds}

An n-dimensional manifold $\mathcal{M}$ is a topological space $(\mathcal{M}, \Theta)$ with the property that each point $p \in \mathcal{M}$ has a 
neighborhood that is homeomorphic to the Euclidean space $\mathbb{R}^{n}$. Thus, if $\forall p \in \mathcal{M}, \exists \mathcal{U} \in \Theta \mid \sigma: \mathcal{U} \mapsto \sigma(\mathcal{U}) \subset \mathbb{R}^{n}$ for which the following conditions hold:

$$
\begin{aligned}
& \sigma \text { is invertible, thus } \exists \sigma^{-1}: \sigma(\mathcal{U}) \mapsto \mathcal{U} \\
& \sigma \text { is continuous } \\
& \sigma^{-1} \text { is continuous }
\end{aligned}
$$

Then $(\mathcal{U}, \sigma)$ is called a chart at $(\mathcal{M}, \Theta)$ and $\sigma: \mathcal{U} \mapsto \sigma(\mathcal{U}) \subset \mathbb{R}^{n}$ is called a chart map.

Although there are different classifications of manifolds, differentiable manifolds are of primary focus along this work, because this type of manifold allows a globally differentiable tangent space, shown in Fig. 3, to be defined using calculus. For each point $p \in \mathcal{M}$, the tangent space $T_{p} \mathcal{M}$ is the space formed by the collection of all tangent vector velocities that a curve $\gamma(t)$ passing through $p$ may have. More formal definitions and a more detailed introduction of the tangent space can be found in [18].

\section{B. Surfaces}

Considered smooth surfaces embedded in $\mathbb{R}^{3}$, which in practice would cover almost all encountered vessel surfaces, are 2-Dimensional parallelizable manifolds $\mathcal{M}=\left\{(x, y, z) \in \mathbb{R}^{3} \mid \phi(x, y, z)=0\right\}$, where $\phi: \mathbb{R}^{3} \rightarrow \mathbb{R}$ is a scalar function that imposes a constraint that defines the shape of the surface. A manifold is called parallelizable if there exists a smooth vector field $\left\{B_{1}, B_{2}\right\}$, such that for every point $p \in \mathcal{M}$, the tangent vectors $\left\{B_{1}(p), B_{2}(p)\right\}$ provide a basis of the tangent space $T_{p} \mathcal{M}$ at $p$. Within these surfaces being considered are explicit surfaces, where one of its variables can be solved for given the constraint imposed by $\phi(x, y, z)=0$ (e.g. a paraboloid), and implicit surfaces which are described by an implicit equation $\phi(x, y, z)$, where one of its variables cannot be solved for (e.g. a cylinder). However, any given surface embedded in $\mathbb{R}^{3}$ can always be written in its implicit form $\phi(x, y, z)=0$, where the zeros of the constraint are the points $p \in \mathcal{M}$ of the surface. Therefore, the basis of the tangent space $T_{p} \mathcal{M}$ at $p$, and thus the manifold parallelization, can be defined as follows:

$$
\begin{aligned}
& \mathbf{V}_{1}(p)=\left[\begin{array}{lll}
1 & 0 & \mathrm{D}_{x} \phi(x, y, z)
\end{array}\right]^{T} \\
& \mathbf{V}_{2}(p)=\left[\begin{array}{lll}
0 & 1 & \mathrm{D}_{y} \phi(x, y, z)
\end{array}\right]^{T}
\end{aligned}
$$

Although this way of defining the parallelization is perfectly valid, it is not the only admissible one, and, as shown in Fig. 3, one can also choose a parallelization which forms an orthonormal basis of the tangent space $T_{p} \mathcal{M}$ at $p$ :

$$
\begin{aligned}
& \mathbf{L}_{1}(p)=\mathrm{D}_{x} \phi(x, y, z) \mathbf{V}_{2}(p)-\mathrm{D}_{y} \phi(x, y, z) \mathbf{V}_{1}(p) \\
& \mathbf{L}_{2}(p)=\mathrm{D}_{x} \phi(x, y, z) \mathbf{V}_{1}(p)+\mathrm{D}_{y} \phi(x, y, z) \mathbf{V}_{2}(p) \\
& \mathbf{B}_{1}(p)=\frac{L_{1}(p)}{\left\|L_{1}(p)\right\|} \quad \mathbf{B}_{2}(p)=\frac{L_{2}(p)}{\left\|L_{2}(p)\right\|}
\end{aligned}
$$

Then, the normal vector to the tangent space $T_{p} \mathcal{M}$ can be

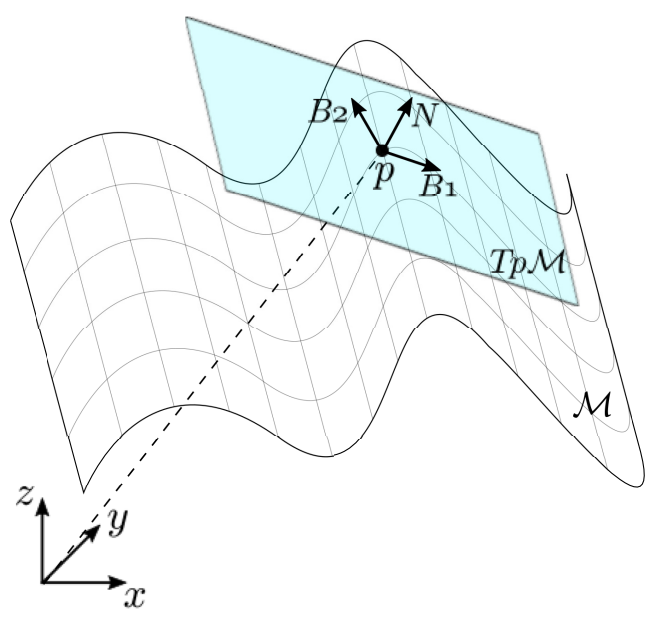

Fig. 3. Illustration of a manifold $\mathcal{M}$, the tangent space $T_{p} \mathcal{M}$ at $p \in \mathcal{M}$ and its basis vectors $\left\{B_{1}(p), B_{2}(p), N(p)\right\}$.

computed at $p$ as follows:

$$
\begin{aligned}
\mathbf{L}_{3}(p) & =\left[\begin{array}{lll}
\mathrm{D}_{x} \phi(x, y, z) & \mathrm{D}_{y} \phi(x, y, z) & -1
\end{array}\right]^{T} \\
\mathbf{N}(p) & =\frac{\mathbf{L}_{3}(p)}{\left\|\mathbf{L}_{3}(p)\right\|}
\end{aligned}
$$

Furthermore, for any given surface, or in other words, for any considered manifold, we can choose a chart and hence a continuous, differentiable, and invertible chart map $\sigma: \mathcal{M} \rightarrow \mathbb{R}^{2}$ which maps points from the manifold to a euclidean space of a dimension equal to $\operatorname{dim}(\mathcal{M})$.

\section{Spline Interpolation}

Bivariate b-splines, which are piecewise polynomial functions can fit a variety of complex shapes while maintaining continuity in their derivatives. This surface representation can be evaluated at any point, and being a polynomial, the derivatives are easily obtained, making it sufficient to create a basis for the tangent space so that the manifold properties and constraints can be applied in the state estimation. The surface is defined as follows:

$$
f(x, y)=\sum_{i=1}^{k} \sum_{j=1}^{l} B_{x i} B_{y j} c_{i j}
$$

The coefficients $c_{i j}$ are determined from the vertices being interpolated. The b-splines $B_{x}$ and $B_{y}$ are determined from their endpoints, known as knots, in each respective dimension, for each piecewise polynomial. Then, the coefficients are multiplied by the tensor product of the b-splines resulting in a surface [19].

\section{METHODOLOGY}

In this section, the general problem of state estimation for a wheeled robot moving on a smooth surface and a detailed description of the adopted methodology to solve this problem is introduced, followed by the experimental procedure that was carried out. This includes the process of charting the manifold and applying an M-IEKF to a minimal state represented on the product space between the chosen chart and $S O(2)$, or directly on $S E(2)$. 


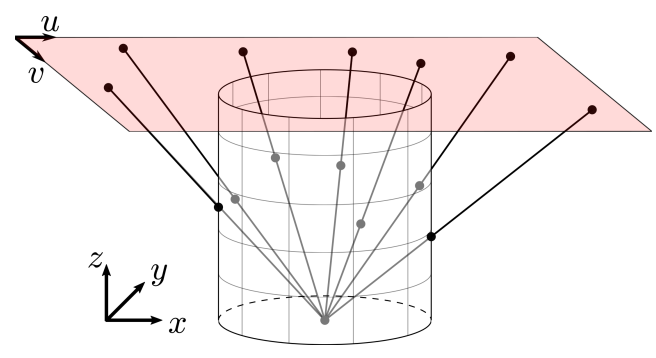

Fig. 4. Illustration of stereographic projection leveraged to define a continuous, differentiable and invertible chart map on the cylinder.

The key to implementing the methodology is to first find a chart that covers the whole manifold being considered, and hence find a continuous, differentiable chart map $\sigma(p)$, and its inverse $\sigma^{-1}(u, v)$.

Let us first consider the easiest case of an explicit, smooth surface described by an explicit function where one of the variables involved is solved for. For example, $z=f(x, y)$. In this case, for every $p \in \mathcal{M}$, the chart map and its inverse are simply determined as follows:

$$
\begin{aligned}
& \sigma(p)=\sigma(x, y, z)=\left[\begin{array}{l}
u \\
v
\end{array}\right]=\left[\begin{array}{l}
x \\
y
\end{array}\right] \\
& \sigma^{-1}(u, v)=p=\left[\begin{array}{l}
x \\
y \\
z
\end{array}\right]=\left[\begin{array}{c}
u \\
v \\
f(u, v)
\end{array}\right]
\end{aligned}
$$

In the most difficult case of implicit, smooth surfaces, a chart, and hence a chart map covering the whole manifold needs to be defined without having a simple and predefined recipe to apply. Consider a cylinder of radius $R$ and height $h$ as a possible manifold to cover with a chart. As a first solution, mapping every point of the cylinder to a plane by unwrapping the cylinder seems logical, however, this solution will result in discontinuities at the border of the map at $2 \pi$. Instead, the stereographic projection can be leveraged to find a continuous, differentiable chart map, shown in Fig. 4, and defined as follows:

$$
\begin{gathered}
\sigma(p)=\sigma(x, y, z)=\left[\begin{array}{l}
u \\
v
\end{array}\right]=\left[\begin{array}{c}
\frac{x h}{\exp (z)} \\
\frac{y h}{\exp (z)}
\end{array}\right] \\
\sigma^{-1}(u, v)=\left[\begin{array}{l}
x \\
y \\
z
\end{array}\right]=\left[\begin{array}{c}
\frac{R u}{\sqrt{u^{2}+v^{2}}} \\
\frac{R v}{\sqrt{u^{2}+v^{2}}} \\
\log \left(\frac{R h}{\sqrt{u^{2}+v^{2}}}\right)
\end{array}\right]
\end{gathered}
$$

Once a chart covering the manifold has been found, an IEKF is applied on a space which is partially defined by the chosen chart and then lifted back to all the estimated results on the manifold. In order to do so, first, a mapping that allows us to map a velocity vector (or displacement vector) $[\Delta b 1 \Delta b 2]^{T}$ on the tangent space $T_{p} \mathcal{M}$ at $p$ to a velocity vector (or displacement vector) $[\Delta x \Delta y \Delta z]^{T}$ on $\mathbb{R}^{3}$ must be found. Then, the chosen chart map must be used to project the robot position to the chart. In general, if a wheeled robot is moving on a manifold and $p_{k}=\left\{x_{k}, y_{k}, z_{k}\right\} \in \mathcal{M}$ is the position of the robot at a given time step $k$, and $\left[\Delta b_{1 k} \Delta b_{2 k}\right]^{T} \in T_{p} \mathcal{M}$ is the linear displacement vector in the tangent space, then we can compute:

$$
\left[\begin{array}{c}
\Delta x_{k} \\
\Delta y_{k} \\
\Delta z_{k}
\end{array}\right]=\left[\begin{array}{lll}
B_{1}\left(p_{k}\right) & B_{2}\left(p_{k}\right) & N\left(p_{k}\right)
\end{array}\right]\left[\begin{array}{c}
\Delta b_{1 k} \\
\Delta b_{2 k} \\
0
\end{array}\right]
$$

The robot position projected on the manifold can then be easily computed through the chosen chart map as follows:

$$
\left[\begin{array}{l}
u_{k} \\
v_{k}
\end{array}\right]=\sigma\left(x_{k}+\Delta x_{k}, y_{k}+\Delta y_{k}, z_{k}+\Delta z_{k}\right)
$$

It is important to note that if the velocity vector (or dispacement vector) $[\Delta b 1 \Delta b 2]^{T}$ on the tangent space $T_{p} \mathcal{M}$ at $p$ is affected by gaussian noise, the linearity of the mapping in Eq. (16) will allow its gaussianity to be preserved.

If a minimal state representation given by $\mathcal{X}=(\mathbf{t}, \mathbf{R}(\theta))=(u, v, \mathbf{R}(\theta)) \in \mathbb{R}^{2} \times S O(2) \quad$ on the product space between the chosen chart and $S O(2)$, where the rotation defined by $S O(2)$ is the rotation of the robot about its own vertical axis, thus its heading, then an IEKF can be designed following algorithm 1 .

In the case of the ship hull simulation, the same methodology is applied to a bivariate b-spline representation of the surface. The vertices are extracted from the CAD model of the ship and interpolated. In the case of the real metal plate experiment, the vertices are taken from a laser scan of the surface before the experiment is carried out, and the point cloud is interpolated. Fig. 2 shows the magnetic crawler robot attached to the curved metal surface that was used, with a UWB beacon attached to it (a tag), and another in the corner (an anchor). Only one anchor is shown, but In total there were four. The laser was also used during the experiment to track the robot for ground truth. The robot collects four tagto-anchor ranges at a time and uses trilateration to compute its position as a measurement in the update function of the M-IEKF algorithm.

\section{EXPERIMENTS}

\section{A. Evaluation}

In this section, the performance of the M-IEKF is evaluated first by testing it on a cylindrical manifold to show its ability to work with any surface that is a parallelizable manifold and to simulate the case of a cylindrical vessel. The M-IEKF is then compared to a standard filter (MCEKF) that uses two virtual, zero noise measurements to keep the state constrained on the curved surface. Moreover, as a proof of concept for metal structure inspection, we have tested the M-IEKF on a simulated ship hull showing that the proposed methodology can handle the case of a priori not-known surface obtained by bivariate $b$-spline intepolation from known points on the surface. Finally, the real world viability of the M-IEKF in metal structure inspection is shown with an experiement employing a magnetic wheeled crawler robot on a curved metal surface. In this last experiment, the triangulated position of the robot was availabe via UWB measurements.

For the two simulated tests, a Monte-Carlo simulation of $N=100$ trials was run. We computed the Root Mean Squared Error (RMSE) in position and orientation, furthermore, the Average Normalized Estimation Error Squared 


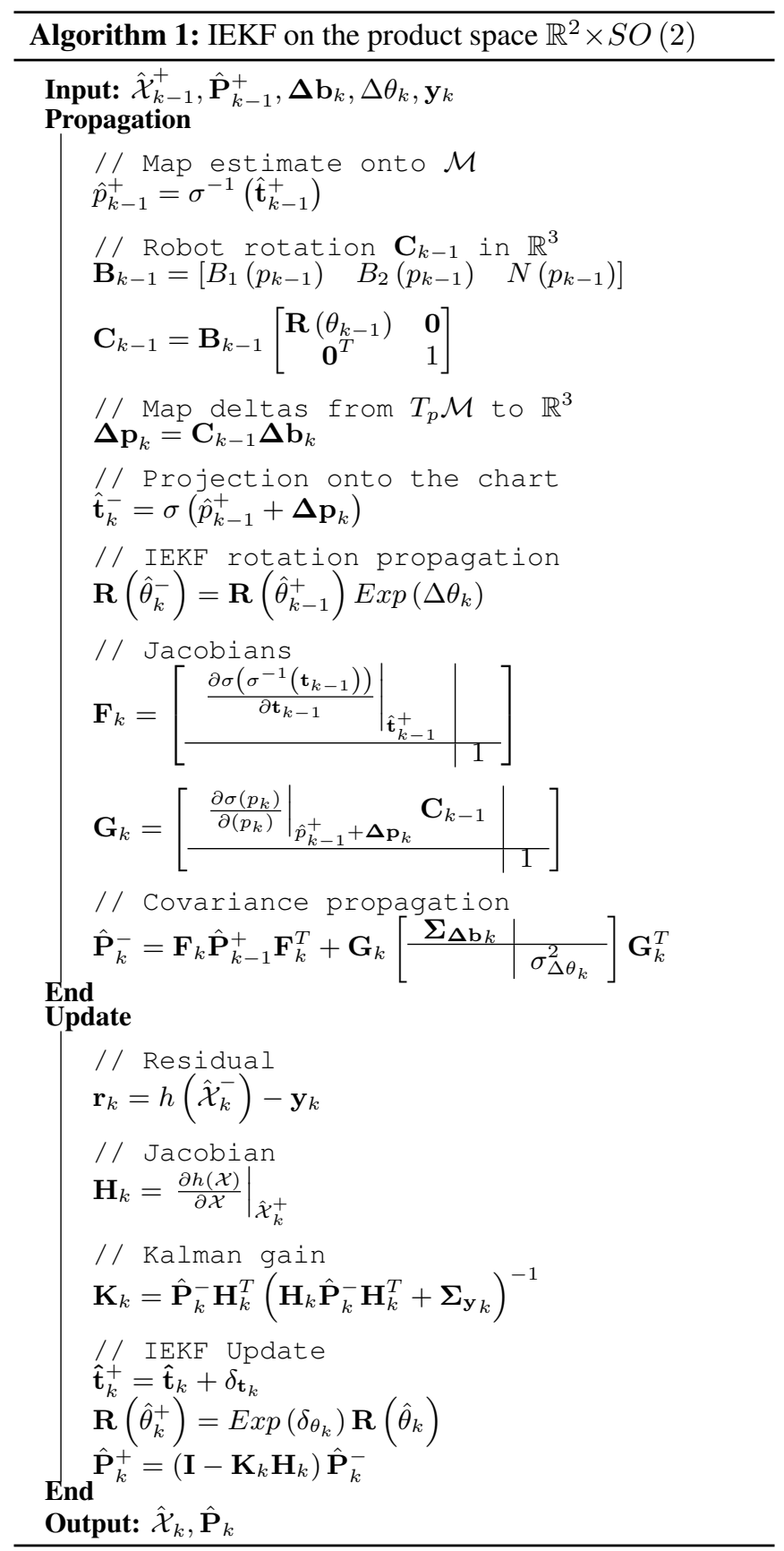

(ANEES) were computed for each time step, averaged over the $N$ trials, and compared between the two filters. The aforementioned metrics are defined as follows:

$$
\begin{gathered}
R M S E=\sqrt{\frac{\sum_{i=1}^{N} \mathbf{e}_{i_{k}}^{2}}{N}} \\
A N E E S=\frac{1}{N m} \sum_{i=1}^{N} \mathbf{e}_{i_{k}}^{T} \mathbf{P}_{i_{k}}^{-1} \mathbf{e}_{i_{k}}
\end{gathered}
$$

where $\mathbf{e}_{i_{k}}$ and $\mathbf{P}_{i_{k}}$ are respectively the estimation error and the error covariance for the $i$-th run at a given time step $k$.

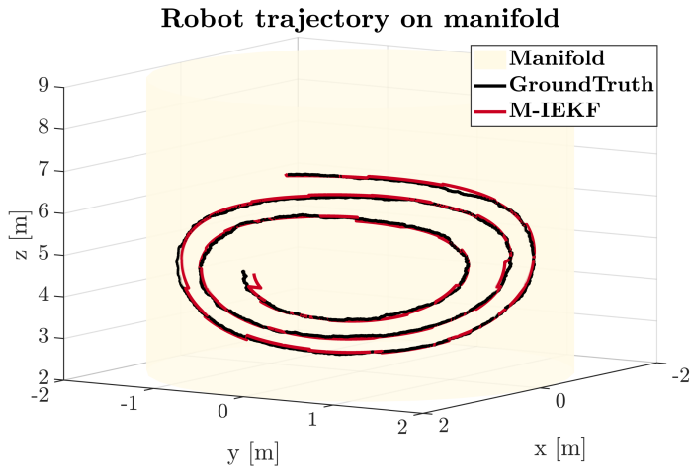

Fig. 5. Ground-truth (in black) and estimated trajectory (in red) of the M-IEKF on a cylindrical surface. Note the wrong initialization of the filter.

M-IEKF position, heading RMSE and pose ANEES

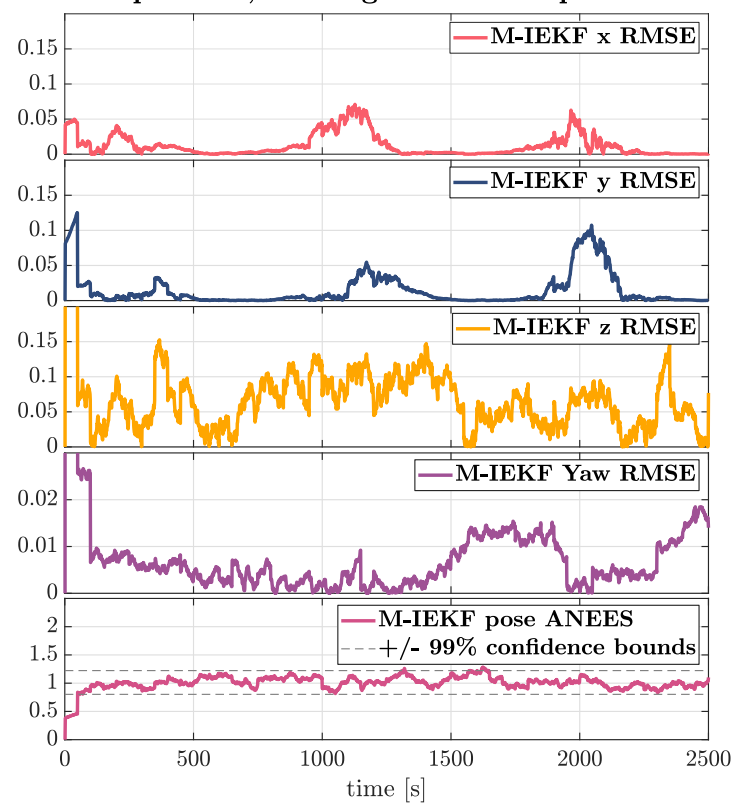

Fig. 6. M-IEKF full state RMSE and ANEES averaged over 100 runs corresponding to the estimation problem on the cylindrical surface.

The RMSE gives an indication of how far the estimate varies from the ground truth on average, whereas the ANEES, which is normalized by the covariance of the filter at each time step, gives a standard for whether a filter is a credible estimator. The closer to 1 an estimator is within the probability interval, the more credible it is, and therefore the more consistency the filter has [20] [21].

\section{B. Results}

Fig. 5 and 6 show the trajectory and the error metrics respectively for the M-IEKF during the cylinder manifold simulation. The trajectory plot shows that the state estimate follows closely with the ground truth which is also corroborated by the error metrics. The RMSE for the heading is mostly below $0.01 \mathrm{rad}$, and the RMSE for its position are predominantly below $10 \mathrm{~cm}$ in each dimension giving a good indication that the filter can perform accurately. Furthermore, the ANEES is almost completely confined to the probability interval, and it is centered about 1 indicating that the filter is credible and consistent. To further evaluate 


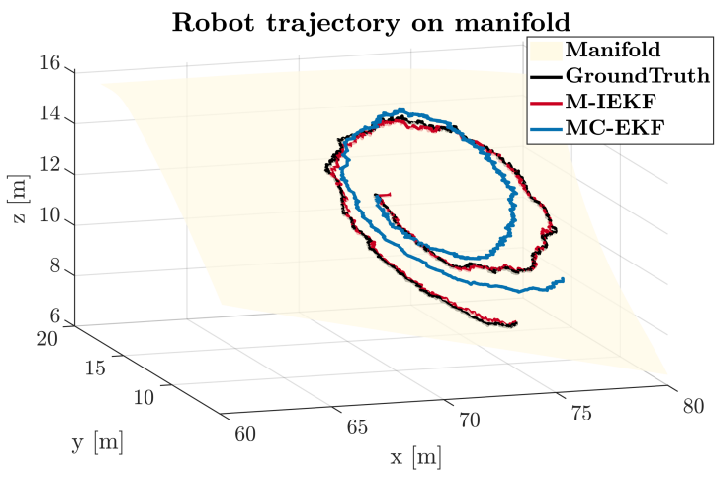

Fig. 7. Ground-truth (in black) and estimated trajectory of the M-IEKF and the MC-EKF (respectively in red and blue) on a b-spline interpolated surface corresponding to the curved surface of a ship hull.

the filter, Fig. 7 and 9 show the trajectory and the error metrics respectively for the M-IEKF and the MC-EKF during the ship hull simulation. The trajectory plot shows that the state estimate of the M-IEKF follows closely with the ground truth like it did in the cylinder experiment, whereas the MCEKF clearly starts diverging. The error metrics show that the M-IEKF still performs consistently and accurately, but with a little bit more error in comparison with the error in the cylinder simulation which was expected considering that its state is being estimated on an interpolated surface this time. By contrast, the MC-EKF shows significantly higher error in the RMSE for its position up to $50 \mathrm{~cm}$ in some instances in the $\mathrm{x}$ direction, and the ANEES plot clearly shows that it goes outside of the probability interval and is therefore not consistent. Fig. 8 shows the trajectory from the real experiment on the curved metal plate for each filter along with the UWB measurements, and Fig. 10 shows the position RMSE for each filter. The M-IEKF follows quite closely to the ground truth, only having noticeable error when there is a high concentration of erroneous UWB measurements due to the metal surface deflection which can be seen near time step 625 . The MC-EKF does not follow closely to the ground truth as expected with errors up to $80 \mathrm{~cm}$. The results back up the fact that the M-IEKF is consistent and more accurate than standard approaches like the MC-EKF, allowing further extensions like the inclusion of a measurement update rejection test, making it a viable option for consistent and robust metal structure inspection with ultra-wideband localization.

\section{CONCLUSION}

The Manifold Invariant Extended Kalman Filter is a novel approach for consistent state estimation on manifolds. It combines manifold state representation and invariance to achieve greater consistency and accuracy. We proved that the proposed M-IEKF is applicable to a wide range of vessel surfaces encountered in real world applications. Further, we showed results validating that the M-IEKF outperforms classical approaches when using real robot wheel odometry and UWB measurements. Therefore, the M-IEKF makes metal structure inspection with ultra-wideband localization viable.

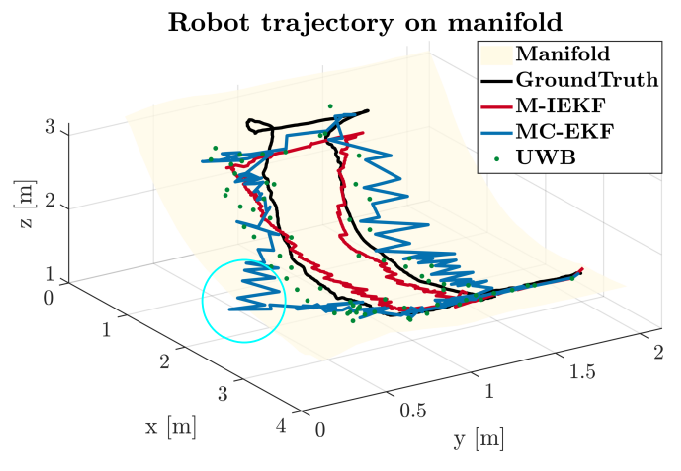

Fig. 8. Ground-truth of the magnetic crawler robot (in black) and estimated trajectory of the M-IEKF and the MC-EKF (respectively in red and blue). Dots (in green) correspond to the position measurements from the UWB trilateration. Note the cyan circle showing the failure of the MC-EKF on providing an estimate that is not attached to the surface.

\section{M-IEKF, MC-EKF position RMSE and ANEES}
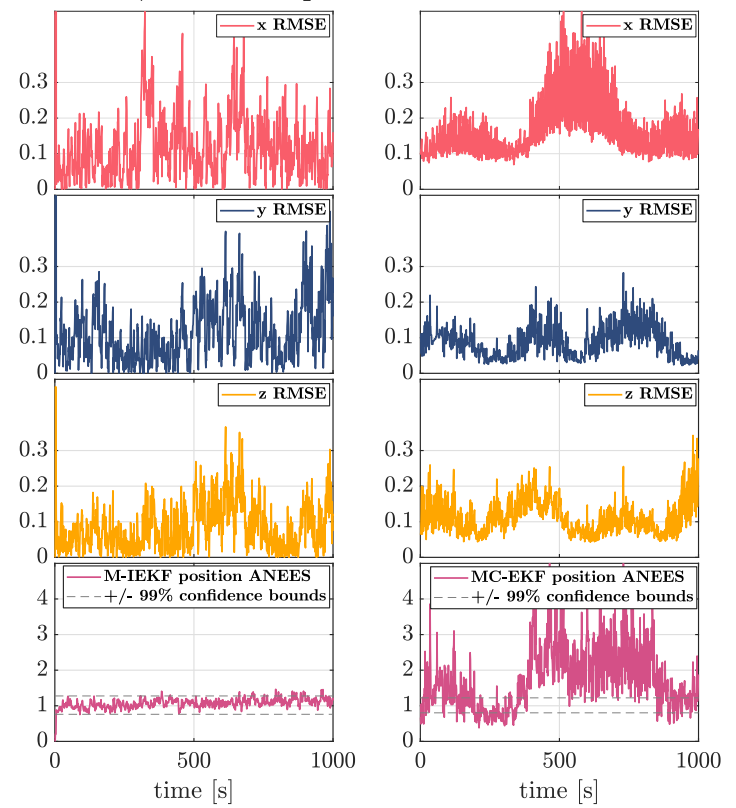

Fig. 9. Comparison between MC-EKF and M-IEK in terms of position RMSE and ANEES corresponding to the case of b-spline interpolated surface.

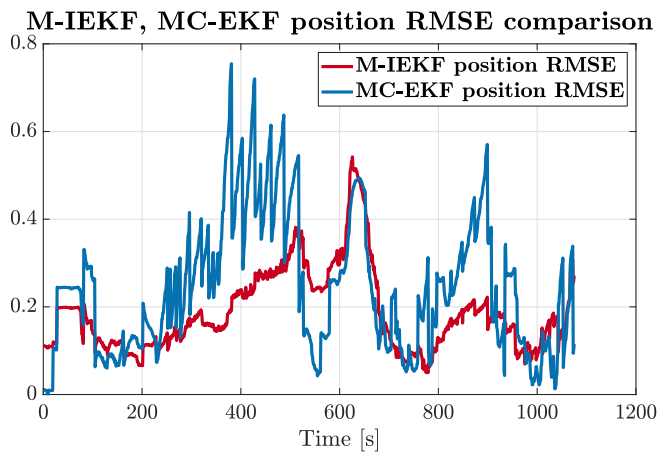

Fig. 10. Position RMSE of the M-IEKF and MC-EKF (in red and blue respectively) corresponding to the real magnetic crawler robot experiment. 


\section{REFERENCES}

[1] A. Barrau and S. Bonnabel, "The invariant extended kalman filter as a stable observer," IEEE Transactions on Automatic Control, vol. 62, no. 4, pp. 1797-1812, 2016.

[2] —_, "An ekf-slam algorithm with consistency properties," arXiv preprint arXiv: $1510.06263,2015$.

[3] - "Invariant kalman filtering," Annual Review of Control, Robotics, and Autonomous Systems, vol. 1, no. 1, pp. 237-257, 2018. [Online]. Available: https://doi.org/10.1146/annurev-control-060117105010

[4] E. Allak, A. Fornasier, and S. Weiss, "Consistent covariance preintegration for invariant filters with delayed measurements," in 2020 IEEE/RSJ International Conference on Intelligent Robots and Systems (IROS). IEEE, 2020.

[5] S. Thrun, "Probabilistic robotics," Communications of the ACM, vol. 45 , no. 3 , pp. $52-57,2002$.

[6] M. Mirbach, "A simple surface estimation algorithm for uwb pulse radars based on trilateration," in 2011 IEEE International Conference on Ultra-Wideband (ICUWB). IEEE, 2011, pp. 273-277.

[7] D. Gao, A. Li, and J. Fu, "Analysis of positioning performance of uwb system in metal nlos environment," in 2018 Chinese Automation Congress (CAC). IEEE, 2018, pp. 600-604.

[8] A. J. Trevor, J. G. Rogers, C. Nieto, and H. I. Christensen, "Applying domain knowledge to slam using virtual measurements," in 2010 IEEE International Conference on Robotics and Automation. IEEE, 2010, pp. 5389-5394.

[9] S. Jung, S. Song, S. Kim, J. Park, J. Her, K. Roh, and H. Myung, "Toward autonomous bridge inspection: A framework and experimental results," in 2019 16th International Conference on Ubiquitous Robots (UR). IEEE, 2019, pp. 208-211.

[10] M. S. B. M. Soberi and M. Z. B. Zakaria, "Autonomous ship hull inspection by omnidirectional path and view," in 2016 IEEE/OES Autonomous Underwater Vehicles (AUV). IEEE, 2016, pp. 38-43.

[11] S. Lan, C. Yang, B. Liu, J. Qiu, and A. Denisov, "Indoor real-time multiple moving targets detection and tracking using uwb antenna arrays," in 2015 International Symposium on Antennas and Propagation (ISAP). IEEE, 2015, pp. 1-4.

[12] R. Zetik, O. Hirsch, and R. Thoma, "Kalman filter based tracking of moving persons using uwb sensors," in 2009 IEEE MTT-S International Microwave Workshop on Wireless Sensing, Local Positioning, and RFID. IEEE, 2009, pp. 1-4.

[13] L. Cheng, H. Chang, K. Wang, and Z. Wu, "Real time indoor positioning system for smart grid based on uwb and artificial intelligence techniques," in 2020 IEEE Conference on Technologies for Sustainability (SusTech). IEEE, 2020, pp. 1-7.

[14] J. Clemens and K. Schill, "Extended kalman filter with manifold state representation for navigating a maneuverable melting probe," in 2016 19Th International Conference On Information Fusion (FUSION). IEEE, 2016, pp. 1789-1796.

[15] H. Benzerrouk and A. Nebylov, "Robust imu/uwb integration for indoor pedestrian navigation," in 2018 25th Saint Petersburg International Conference on Integrated Navigation Systems (ICINS). IEEE, 2018, pp. 1-5.

[16] B. B. Ready, "Filtering techniques for pose estimation with applications to unmanned air vehicles," 2012.

[17] M. Brossard, A. Barrau, and S. Bonnabel, "A code for unscented kalman filtering on manifolds (ukf-m)," arXiv preprint arXiv:2002.00878, 2020.

[18] P.-A. Absil, R. Mahony, and R. Sepulchre, Optimization algorithms on matrix manifolds. Princeton University Press, 2009.

[19] C.-J. Li and R.-H. Wang, "Bivariate cubic spline space and bivariate cubic nurbs surfaces," in Geometric Modeling and Processing, 2004. Proceedings. IEEE, 2004, pp. 115-123.

[20] X. R. Li, Z. Zhao, and V. P. Jilkov, "Practical measures and test for credibility of an estimator," in Proc. Workshop on Estimation, Tracking, and Fusion-A Tribute to Yaakov Bar-Shalom. Citeseer, 2001, pp. 481-495.

[21] X. R. Li, Z. Zhao, and X. B. Li, "Evaluation of Estimation Algorithms: Credibility Tests," IEEE Transactions on Systems, Man, and Cybernetics Part A: Systems and Humans, vol. 42, no. 1, pp. 147-163, 2012. 